\title{
Multiple brain abscesses treated with antibiotics: Report of a case
}

\author{
CONNIE JO MCCARROLL, DO \\ H. LAUREN VOGEL, DO
}

In recent years some cases of brain abscesses have been treated successfully with antibiotics but not neurosurgery. Such cases include those with smaller or multiple lesions that are surgically inaccessible in patients without severe neurologic deficits. The antibiotics must be specific for the offending organism and able to cross the blood-brain barrier. CAT scanning is important to follow the progress of the case. If conditions warrant, neurosurgical intervention must be considered. A case of Staphylococcus sepsis complicated by endocarditis and multiple brain abscesses provides an illustration of nonsurgical management.

The mortality from brain abscesses has declined since utilizing computerized axial tomography (CAT) scanning has permitted early diagnosis. The mortality from 1958-1972 ranged from $27 \%$ to $53 \%,{ }^{1}$ but more recent studies have indicated a better prognosis with rates from $5 \%$ to $10 \%{ }^{2}$ Also the therapeutic approach to brain abscesses has changed. This change has been brought about by the knowledge that some brain abscesses can be treated successfully with antibiotics. In 1986 Rosenblum and coauthors ${ }^{3}$ found reports in the literature of 67 cases treated without surgical intervention. In 53 patients whose neurologic status at hospital admission and discharge was known, all 53 were alert with slight or no deficits at hospital discharge.

Recent experience with a case of staphylococcal sepsis complicated by endocarditis and multiple brain abscesses provides an opportunity to explore the role of antibiotic therapy and follow-up with CAT scanning in the management of brain abscesses.

\section{Report of case}

A 2 1/2-year-old boy was admitted to the pediatric unit from the emergency department with abdominal pain and fever of 24 hours'duration. He had been seen in the emergency department two to three days before because he had a piece of glass in his left foot; no treatment had been given. In the emergency department at the time of admission the pulse rate was 186 beats per minute, respirations were $24 / \mathrm{min}$, and the temperature was $102.2^{\circ} \mathrm{F}$; the blood pressure had not been measured.

On admission to the Pediatric Intensive Care Unit, the blood pressure was $84 / 60 \mathrm{~mm} \mathrm{Hg}$, pulse, 128 beats per minute, respirations, $44 / \mathrm{min}$, and temperature, $104.6^{\circ} \mathrm{F}$. Eye examination revealed neither Roth spots or petechiae. Pupils were equal and reactive to light and accommodation. The extraocular muscles were intact. The neck was supple. No meningeal irritation was evident. Cardiorespiratory examination revealed grunting respirations. The lungs were clear to auscultation. The heart rate was increased with a regular rhythm. No murmurs were auscultated. The abdomen was diffusely tender with exquisite tenderness in the right lower quadrant. The liver and spleen were not enlarged. The child was alert but irritable. No papilledema was found. Deep tendon reflexes were normal, Babinski's reflexes were down going, and Kernig's and Brudzinski's signs were absent. The skin was warm with no petechiae or nodules. No masses were detected. An abscess with a possible foreign body was noted in the left foot.

Initial laboratory studies revealed the following values: WBC, $17 \times 10^{3} / \mu \mathrm{L} ; 76 \%$ segmented forms, $13 \%$ band cells, 9 lymphocytes, and 2 monocytes; hemoglobin, 13.6 $\mathrm{g} / \mathrm{dL}$; hematocrit, $40 \%$; platelets, $389,000 / \mu \mathrm{L}$. No toxic granulations were noted. Quantitative C-reactive protein (CRP) was $0.2 \mathrm{mg} / \mathrm{dL}$. Electrolyte values were as follows: sodium, $127 \mathrm{mEq} / \mathrm{L}$; potassium, $6.2 \mathrm{mEq} / \mathrm{L}$; chloride, $102 \mathrm{mEq} / \mathrm{L}$; and $\mathrm{CO}_{2}, 11 \mathrm{mEq} / \mathrm{L}$. The $\mathrm{BUN}$ was 9 $\mathrm{mg} / \mathrm{dL}$. Urinalysis showed $2+$ glucose and granular casts. Material from the left foot lesion and blood were taken for culture and sensitivity testing.

Following admission to the Pediatric Intensive Care Unit, the blood pressure decreased to $80 / 36 \mathrm{~mm} \mathrm{Hg}$, the respiratory rate increased to $68 / \mathrm{min}$, and the heart rate increased to 200 . Capillary refill was greatly diminished. Shock was treated successfully with fluid resuscitation using $400 \mathrm{cc} / \mathrm{m}^{2}$ Ringer's lactate solution over a 20 minute-period. Surgical consultation to evaluate the abdomen as the site responsible for the shock ruled out a surgical abdomen.

The child became more stuporous and had a generalized seizure. He was given diazepam (Valium) $4 \mathrm{mg}$ intravenously followed by $50 \mathrm{mg}$ phenobarbital slowly. Respiratory status was monitored closely. Phenobarbital administration, $30 \mathrm{mg}$ every 12 hours, was continued. Re- 

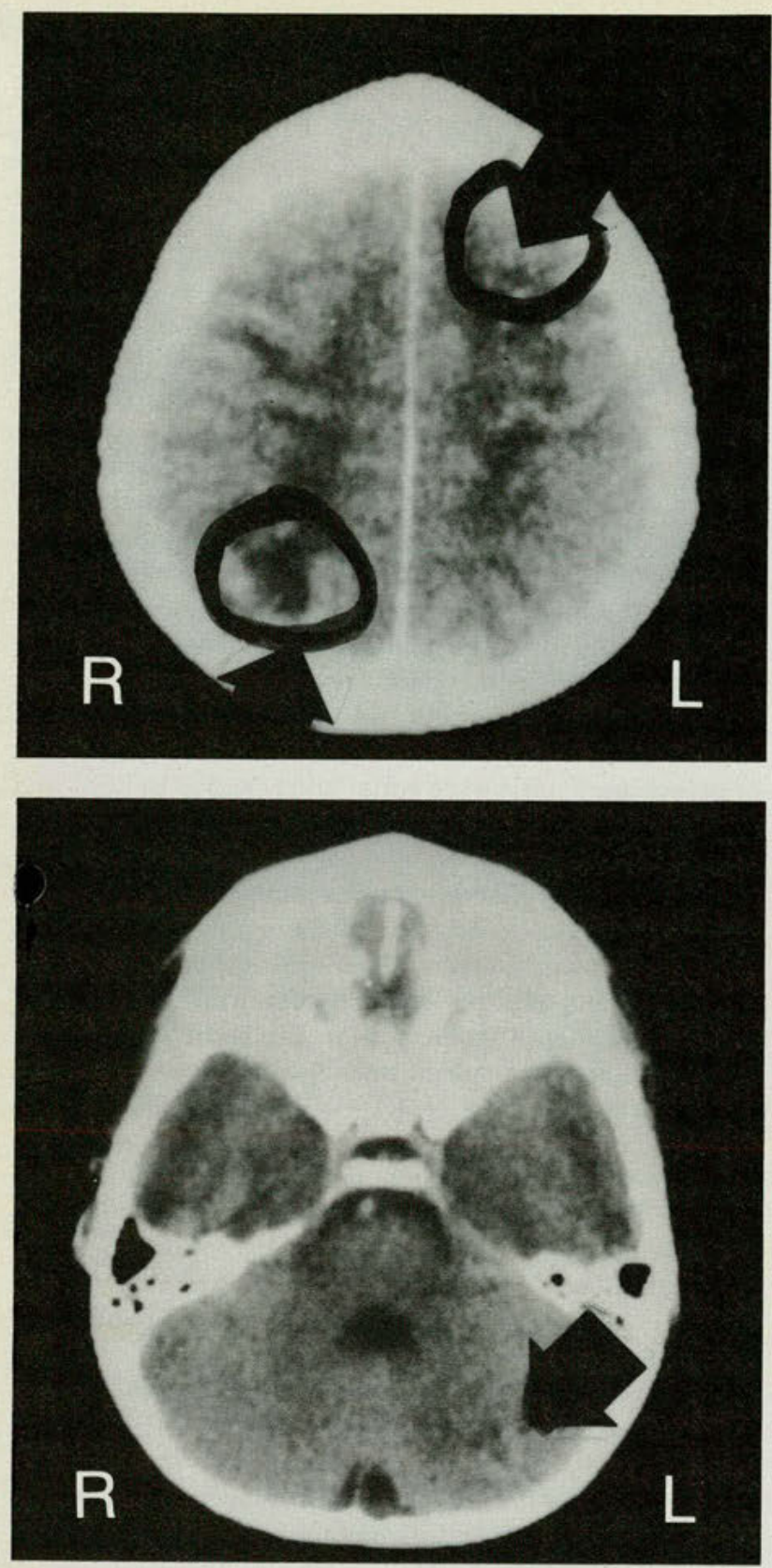

Figs 1-2 Initial CAT scan after focal left-sided seizure. Abscess formation left and right cerebral hemispheres and left cerebellum indicated by hypodense areas with rim enhancement.

sults of a lumbar puncture performed at this time were negative. Cerebrospinal fluid and urine testing for latex agglutination against Hemophilus influenzae, Pneumococcus, and Neisseria menigitidis yielded negative results. The child received chloramphenicol sodium succinate, $100 \mathrm{mg} / \mathrm{kg} /$ day, and ampicillin sodium, $200 \mathrm{mg} / \mathrm{kg} /$ day, while culture results were awaited.

Approximately 12 hours after admission, the temperature spiked to $105^{\circ} \mathrm{F}$. At this time a midsystolic murmur grade $2 / 6$ was detected; the murmur increased to holosystolic grade $3 / 6$, over the next two hours. No eye or skin lesions were noted. Staphylococcus aureus was

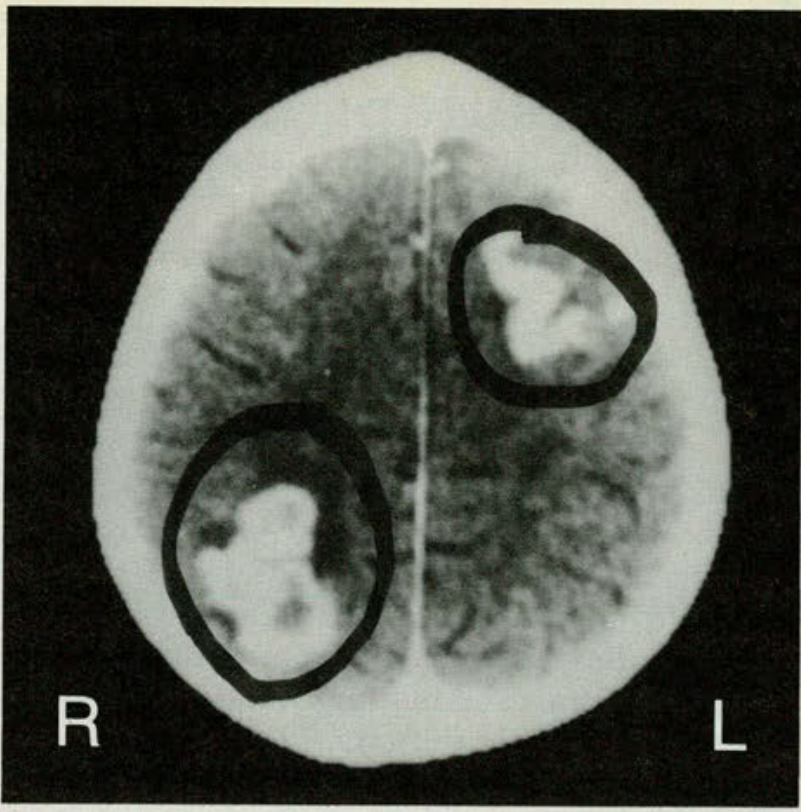

Figs 3-4 CAT scan two weeks later showing worsening of the cerebral hemispheres and cerebellar abscesses.

cultured from both the blood and the left foot. Antibiotics were changed to oxacillin sodium $200 \mathrm{mg} / \mathrm{kg} /$ day and gentamicin sulfate $50 \mathrm{mg} / \mathrm{m}^{2}$ every eight hours. The development of inappropriate antidiuretic hormone syndrome was shown by a serum sodium value of $128 \mathrm{mEq} /$ $\mathrm{L}$, urinary sodium level of $103 \mathrm{mEq} / \mathrm{L}$, and BUN of 9 $\mathrm{mg} / \mathrm{dL}$. With fluid restriction to $800 \mathrm{cc} / \mathrm{m}^{2} /$ day the sodium level normalized.

Temperature spikes continued. Petechiae and subcutaneous nodules were noted. A friction rub was heard over the left sternal border. The quantitative CRP increased to $40 \mathrm{mg} / \mathrm{dL}$. The sedimentation rate was $50 \mathrm{~mm} /$ $\mathrm{hr}$. The cardiac creatine phosphokinase (CPK) was 1,150 . The echocardiogram revealed a small posterior pericardial effusion and multiple echoes on a bicuspid aortic valve consistent with endocarditis. A gallium scan showed cardiac involvement as well as increased uptake in the right knee.

The patient's mental status improved over the next six days. However on day 7 a left-sided focal seizure involving the neck and upper extremity developed. CAT scan of the head showed multiple abscesses in the cerebral hemispheres and the left cerebellum (Figs 1 and 2).

The antibiotic regimen was changed to chloramphenicol palmitate oral suspension, $100 \mathrm{mg} / \mathrm{kg} / \mathrm{day}$, with continuation of oxacillin and discontinuation of gentamicin. Chloramphenicol levels were followed serially, but when toxic level were reached, dosage was decreased to $280 \mathrm{mg} / \mathrm{per}$ dose. The chloramphenicol form was changed to the oral to save precious intravenous sites. The dosage of the oral preparation was $75 \mathrm{mg} / \mathrm{kg} /$ day because it is the active form that, unlike the intravenous preparation, does not require liver metabolism.

CAT scans were followed weekly. During the first two weeks, the cerebral lesions worsened (Figs 3 and 4), but, 

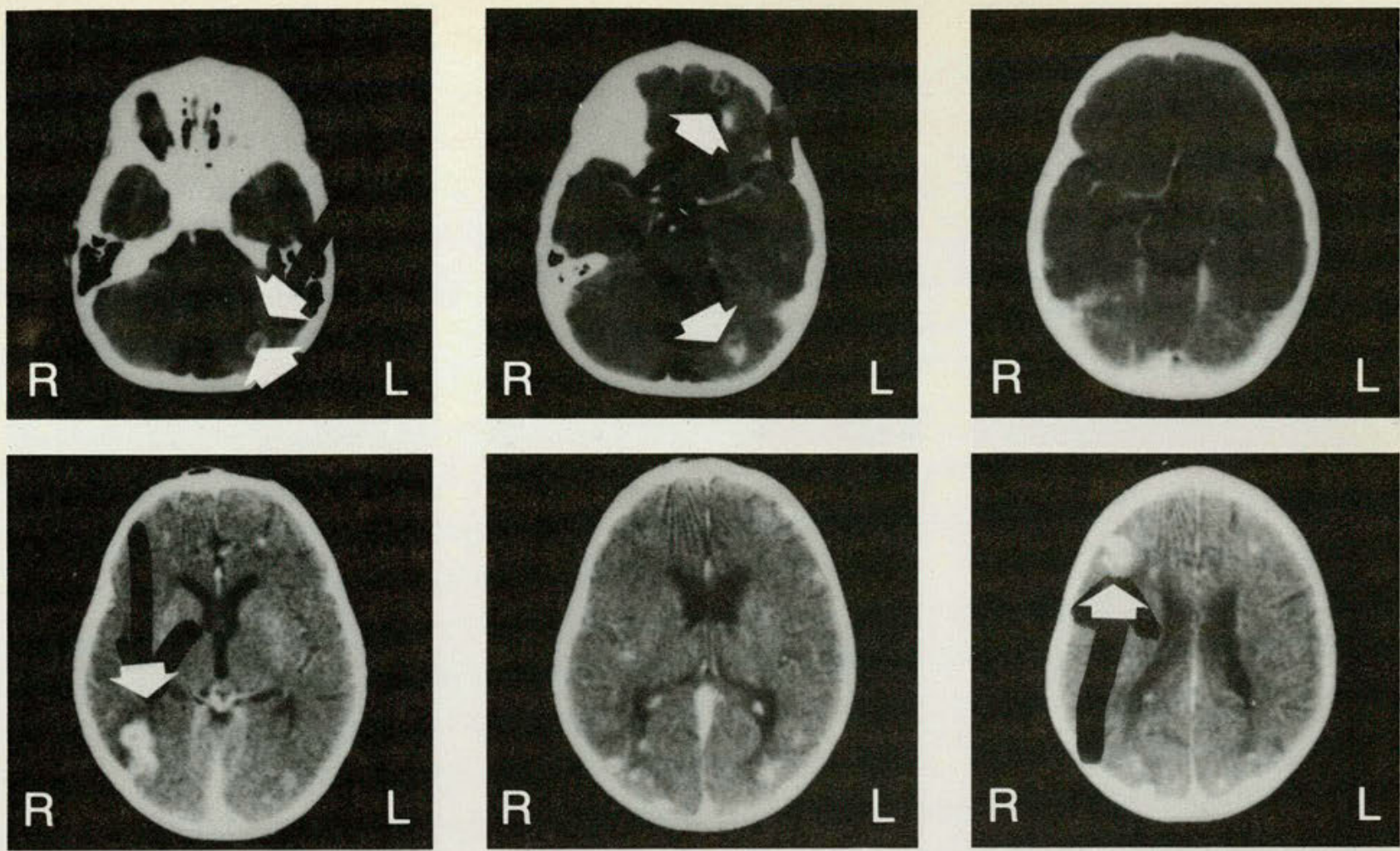

after this, the scans showed resolution of the abscesses (Figs 5 and 6).

During late hospitalization visual difficulties developed. Ophthalmologic examination showed Roth spots as well as pallor of the right inferior temporal quadrant consistent with branch retinal artery occlusion.

The repeat echocardiogram indicated resolution of the aortic valve lesions. The valve again was noted to be bicuspid. Quantitative CRP was followed throughout hospitalization to monitor adequacy of therapy, and the value at discharge was 0.00 (Table 1 ).

The child was discharged on a regimen of chloramphenicol. At discharge he had abnormal, ataxic gait and visual disturbance. Serial CAT scans of the head showed further resolution of the abscesses. $\mathrm{CBC}$ and chloramphenicol levels were determined every two weeks. Chloramphenicol administration was continued for three months. He was enrolled in a program for impaired children but became ineligible after three weeks when no severe impairments remained. A CAT scan 1 year after the first scan showed only a small hypodense area in the right cerebral hemisphere. A recent ophthalmologic examination revealed normal vision and resolution of the branch artery occlusion. The neurologic status is normal at this time. Eighteen months after the initial insult, the child showed no abnormal effects. He was enrolled in a Head Start Program and was performing at a level above that of his peers. At 3-year follow-up, he was neurologically normal and in an advanced kindergarten program.

\section{Review of the literature}

The etiology of brain abscesses includes infection introduced by metastasis, contiguous spread, or trauma. The hematogenous spread may be from distant foci, such as the lower respiratory tract, osteomyelitis, or abdominal wounds. Paranasal sinus disease may cause brain abscess formation by retrograde septic thrombophlebitis to the cerebral cortex or by direct contiguous spread. Abscess formation may follow penetrating wounds or craniotomy. ${ }^{3}$

Staphylococcus aureus and anaerobic or microaerophilic streptococci are the most frequently recovered microorganisms. ${ }^{4}$ Less frequent are Gramnegative aerobic Hemophilus, Escherichia, and Proteus species as well as gram-negative anaerobes such as Bacteroides fragilis. ${ }^{5}$ Isolation of an organism from the CSF fluid or the blood is considered appropriate for identification of the causative agent. ${ }^{6}$ Brain abscesses develop in four stages ${ }^{7}$ : stage 1 , early cerebritis; stage 2 , late cerebritis; stage 3 , early capsule formation; stage 4 , late capsule formation. The time from the implantation of the organism to development of a well-formed capsule is about 14 days. $^{3}$

Brain abscesses should be suspected in a child with headaches, vomiting, an altered state of consciousness, seizures, hemiparesis, visual field de- 

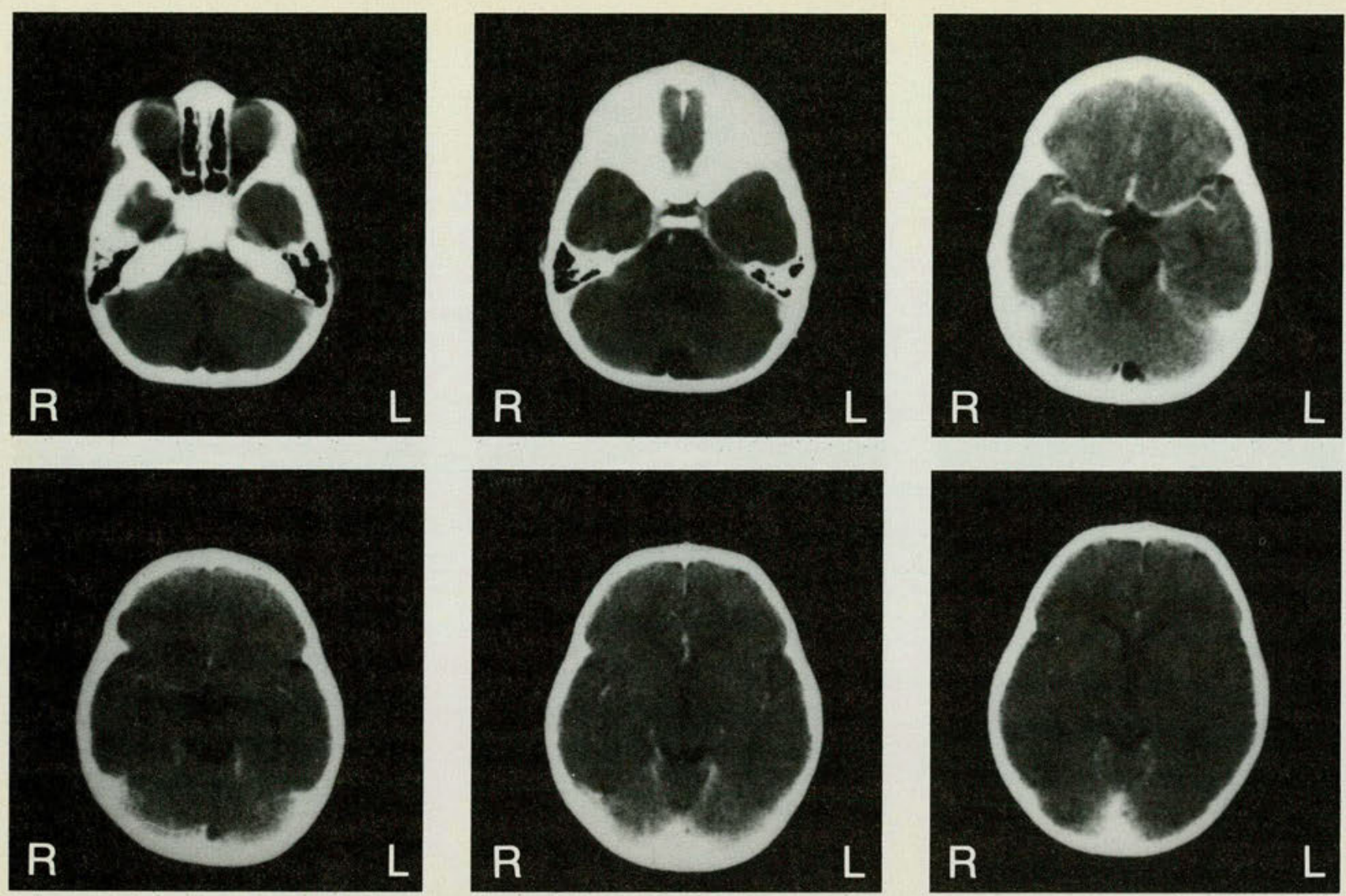

Figs 5-6 CAT scan three months later showing improvement of abscesses with enhancement in right hemisphere.

fects, nuchal rigidity, and papilledema in association with other infectious processes. ${ }^{2}$

Lumbar puncture carries inherent risk in patients with cerebral abscess. Herniation and incarceration have occurred in several children with cerebral abscesses. ${ }^{8}$ Cultures of the blood and other appropriate sites must be obtained. CAT scanning of the head with or without contrast studies must be performed. Although CAT scanning is the diagnostic procedure of choice at this time, magnetic resonance imaging offers promise as well.

The traditional treatment of brain abscesses includes appropriate antibiotic therapy and neurosurgical drainage or excision. ${ }^{9}$ Neurosurgical drainage is not feasible in multiple abscesses, however, unless there is a mass effect secondary to the abscesses. ${ }^{6}$

Successful treatment with appropriate antibiotics may be due to several factors. It has been suggested that intracerebral infective processes are eradicated more readily when therapy is initiated during the cerebritis phase of abscess formation. ${ }^{6}$ Also, small abscesses may be more likely to resolve with antibiotic therapy. Finally, the correct antibiotic at levels that penetrate the brain abscess in sufficient concentrations may determine the response to medical therapy. ${ }^{3}$ Ninety-five percent of abscesses that will resolve with antibiotic therapy will show CAT scan evidence of decreased size after one month of therapy.

A treatment plan has been proposed by Rosenblum and co-workers. ${ }^{3}$ Patients with severe neurologic deficits (grades 3 and 4), abscesses larger than $3 \mathrm{~cm}$ in diameter, or surgically accessible multiple lesions should have the lesions removed or aspirated. Those with better neurologic status (grades 1 or 2) and smaller or multiple lesions may be candidates for antibiotic treatment.

The antibiotic therapy should be specific and bactericidal for the responsible organism. The main concern is to have antibiotics that cross the bloodbrain barrier. ${ }^{3}$

Penicillin and chloramphenicol are appropriate for treating intracerebral abscesses, and, in combination, are effective against most anaerobic and aerobic organisms. If the organism produces penicillinase, then methicillin or other appropriate antibiotics may be added or substituted for penicillin. Dosages of greater than $12 \mathrm{~g}$ daily for methicillin for an adult may be used. In children 200-400 


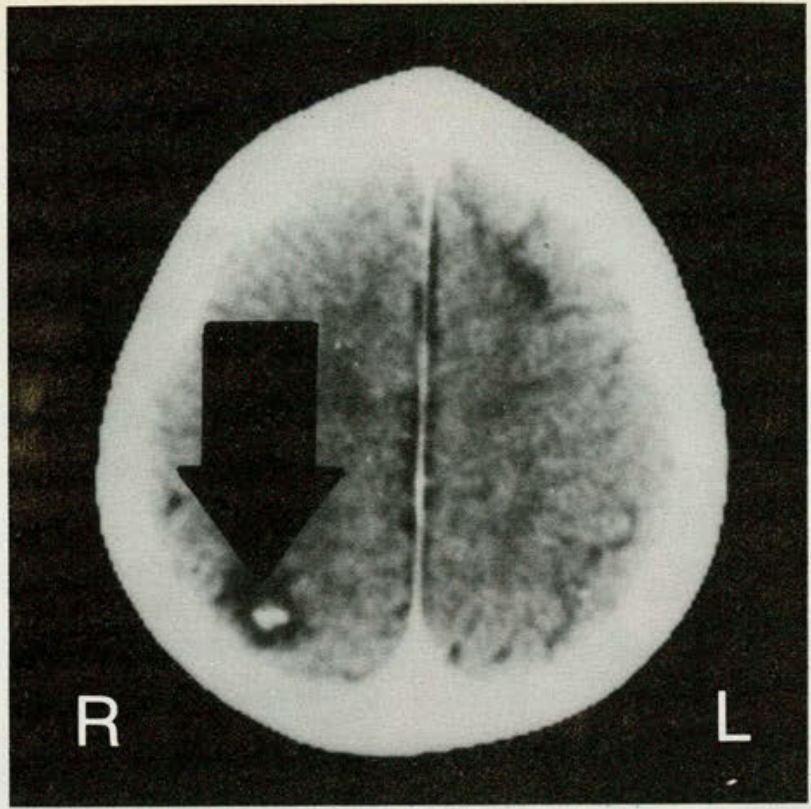

$\mathrm{mg} / \mathrm{kg} /$ day may be used. Ceftriaxone sodium (Rocephin), a third generation cephalosporin, may hold promise because it crosses the blood-brain barrier and is effective against $S$ aureus.

A CAT scan should be performed weekly. If after two weeks, the condition of the patient deteriorates or significant mass effect is noted, surgery should be performed. However, if the patient is improving or stable, antibiotics may be continued for an additonal two weeks. If after the fourth week, the abscess has not improved, then surgery should be performed. Antibiotics should be continued for six to eight weeks. After resolution of the lesion is observed, CAT scans should be performed every two to four months for one year. ${ }^{3}$

Neurosurgical consultation should be obtained early. Intervention must be considered when the patient's condition warrants. ${ }^{6}$

\section{Summary}

In the case reported here, several complications of Staphylococcus sepsis developed: multiple brain abscesses; shock; endocarditis; septic arthritis; and inappropriate antidiuretic hormone syndrome. The patient was treated with antibiotic therapy alone for the brain abscesses, and has continued to improve.

\begin{tabular}{|lc|}
\hline TABLE 1. CRP VALUES DURING HOSPITALIZATION \\
\hline Date & $\begin{array}{c}\text { Quantitative CRP values } \\
(\mathrm{mg} / \mathrm{dL})\end{array}$ \\
\hline $5 / 4$ & 0.22 \\
$5 / 6$ & 40.70 \\
$5 / 8$ & 23.00 \\
$5 / 13$ & 10.80 \\
$5 / 15$ & 9.10 \\
$6 / 2$ & 4.10 \\
$6 / 23$ & 0.80 \\
$6 / 30$ & 0.00 \\
\hline
\end{tabular}

The quantitative CRP was useful in following the adequacy of therapy. It appeared to correlate with the child's recovery.

This case demonstrates that (1) antibiotic therapy alone may be used in the treatment of brain abscesses if no significant deterioration or mass effect develops; and (2) CAT scanning of the head is useful in making an early diagnosis as well as following the course of the abscesses.

1. Whelan MA, Hilal SK: Computed tomography as a guide in the diag nosis and follow-up of brain abscesses. Radiology 1980;135:663-671.

2. Jadavji T, Humphreys RP, Prober CG: Brain abscesses in infants and children. Pediatr Infect Dis 1985;4:394-398.

3. Rosenblum ML, Mampalam TJ, Pons VG: Controversies in the management of brain abscesses. Clin Neurosurg 1986;33:603-632.

4. Idriss ZH, Gutman LT, Kronfol NM: Brain abscesses in infants and children: Current status of clinical findings, management and prognosis. Clin Pediatr 1978;17:738-746.

5. Garvey G: Current concepts of bacterial infections of the central nervous system: Bacterial meningitis and bacterial brain abscess. $J$ Neurosurg 1983;59:735-744.

6. Rosenblum ML, Hoff JT, Norman D, et al: Nonoperative treatment of brain abscesses in selected high-risk patients. $J$ Neurosurg 1980;52:217. 225.

7. Britt RH, Enzmann DR: Clinical stages of human brain abscesses on serial CT scans after contrast infusion: Computerized tomographic, neuropathological, and clinical correlations. J Neurosurg 1983;59:972-989. 8. Nielsen H: Cerebral abscess in children. Neuropediatrics $1983 ; 14$ (May):76-80

9. Keren G, Tyrrell DLJ: Nonsurgical treatment of brain abscesses: Report of two cases. Pediatr Infect Dis 1984;3:331-334.

From the Department of Pediatrics, Grandview Hospital and Medical Center, Dayton, Ohio.

For reprints, address Dr McCarroll, 216 Neal Ave, Dayton, OH 45405 . 


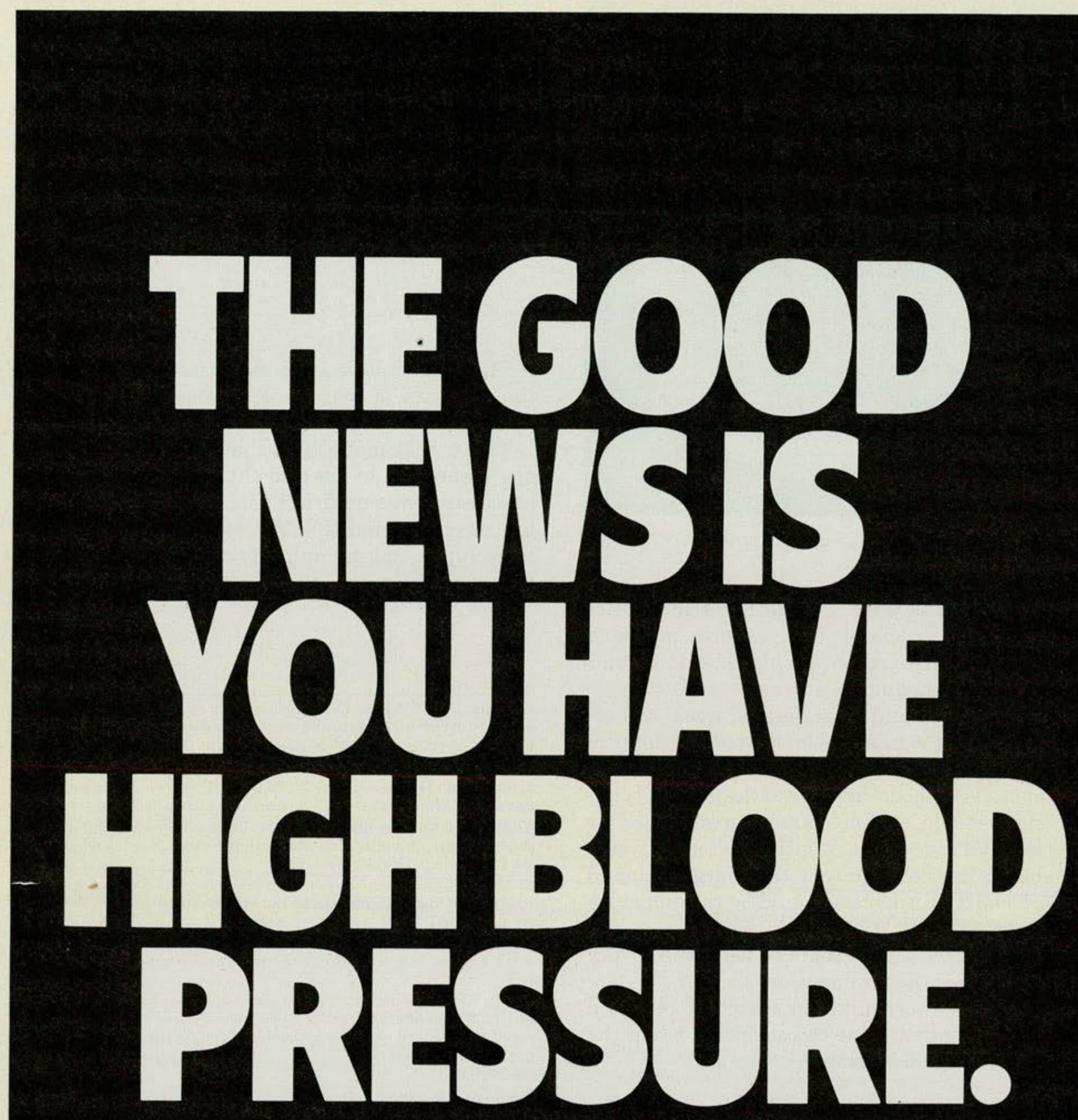

Finding out you've got high blood pressure is half the battle. With a plan for exercise, weight control, salt reduction and medication, the rest is easy.

If you have high blood pressure, get on a good program and stick with it. And do what your doctor says. Then there probably won't be any bad news.

\section{(1) American Heart Association}

